\title{
1 Genetic dissection of an ancient divergence in yeast thermotolerance
}

3 Carly V. Weiss ${ }^{1}$, Jeremy I. Roop ${ }^{1,2 *}$, Rylee K. Hackley ${ }^{1,3,4}$, Julie Chuong ${ }^{3}$,

4 Igor V. Grigoriev ${ }^{1,5}$, Adam P. Arkin ${ }^{6}$, Jeffrey M. Skerker ${ }^{6}$, Rachel B. Brem ${ }^{1,3 *}$

$6{ }^{1}$ Department of Plant and Microbial Biology, UC Berkeley, Berkeley, CA.

$7 \quad{ }^{2}$ Current address: Fred Hutchinson Cancer Research Center, Seattle, WA.

$8 \quad{ }^{3}$ Buck Institute for Research on Aging, Novato, CA.

$9{ }^{4}$ Current address: Duke University Program in Genetics \& Genomics, Duke University, Durham, 10 NC.

$11{ }^{5}$ US Department of Energy Joint Genome Institute, Walnut Creek, CA.

$12{ }^{6}$ Department of Bioengineering, UC Berkeley, Berkeley, CA and Lawrence Berkeley National

13 Laboratory, Berkeley, CA.

14 *Correspondence to: jeremyianroop@gmail.com, rbrem@buckinstitute.org. 


\section{Abstract}

20 Some of the most unique and compelling survival strategies in the natural world are fixed in

21 isolated species. To date, molecular insight into these ancient adaptations has been limited, as

22 classic experimental genetics has focused on interfertile individuals in populations. Here we use

23 a new mapping approach, which screens mutants in a sterile interspecific hybrid, to identify

24 eight housekeeping genes that underlie the growth advantage of Saccharomyces cerevisiae

25 over its distant relative S. paradoxus at high temperature. Pro-thermotolerance alleles at these

26 mapped loci were required for the adaptive trait in S. cerevisiae and sufficient for its partial

27 reconstruction in S. paradoxus. The emerging picture is one in which S. cerevisiae improved the

28 heat resistance of multiple components of the fundamental growth machinery in response to

29 selective pressure. This study lays the groundwork for the mapping of genotype to phenotype in

30 clades of sister species across Eukarya. 
32 Geneticists since Mendel have sought to understand how and why wild individuals differ.

33 Studies toward this end routinely test for a relationship between genotype and phenotype via

34 linkage or association ${ }^{1}$. These familiar approaches, though powerful in many contexts, have an

35 important drawback—they can only be applied to interfertile members of the same species. This

36 rules out any case in which an innovation in form or function evolved long ago and is now fixed

37 in a reproductively isolated population.

39 As organisms undergo selection over long timescales, their traits may be refined by processes

40 quite different from those that happen early in adaptation ${ }^{2,3}$. We know little about these

41 mechanisms in the wild, expressly because when the resulting lineages become reproductively

42 incompatible, classic statistical-genetic methods cannot be used to analyze them ${ }^{4}$. To date, the

43 field has advanced largely on the strength of candidate-based studies that implicate a single

44 variant gene in an interspecific trait ${ }^{5,6}$, with the complete genetic architecture often remaining

45 unknown. Against the backdrop of a few specialized introgression ${ }^{7-10}$ and molecular-evolution ${ }^{11}$

46 techniques available in the field, dissection of complex trait differences between species has

47 remained a key challenge.

49 Here we develop a new genetic mapping strategy, based on the reciprocal hemizygosity 50 test $^{12,13}$, and use it to identify the determinants of a difference in high-temperature growth 51 between isolated Saccharomyces yeast species. We validate the contributions of the mapped 52 loci to the thermotolerance trait, and we investigate their evolutionary history.

\section{Results}

\section{Species differences in thermotolerance}


58 At high temperature, the yeast Saccharomyces cerevisiae grows qualitatively better than other

59 members of its clade ${ }^{14-16}$, including its closest relative, S. paradoxus, from which it diverged $\sim 5$

60 million years ago ${ }^{17}$. In culture at $39^{\circ} \mathrm{C}$, S. cerevisiae doubled faster than S. paradoxus and

61 accumulated more biomass over a timecourse, a compound trait that we call thermotolerance.

62 The magnitude of differences in thermotolerance between species far exceeded that of strain

63 variation within each species (Figure 1), whereas no such effect was detectable at $28^{\circ} \mathrm{C}$ (Figure

$64 \mathrm{~S} 1$ ). The failure by S. paradoxus to grow to high density at $39^{\circ} \mathrm{C}$ was, at least in part, a product

65 of reduced survival relative to that of $S$. cerevisiae, as cells of the former were largely unable to

66 form colonies after heat treatment (Figure S2). In microscopy experiments, S. paradoxus cells

67 were almost uniformly visible as large-budded dyads after 24 hours at $39^{\circ} \mathrm{C}$ (Figure S3),

68 suggestive of defects late in the cell cycle as a proximal cause of death ${ }^{18}$; no such tendency

69 was apparent in S. cerevisiae at high temperature, or in either species at $28^{\circ} \mathrm{C}$ (Figure S3).

71 Massively parallel reciprocal hemizygosity testing by $\mathrm{RH}-\mathrm{seq}$

73 We set out to dissect the genetic basis of $S$. cerevisiae thermotolerance, using a genomic

74 implementation of the reciprocal hemizygote test ${ }^{12,13}$ (Figure 2A). For this purpose, we first

75 mated S. cerevisiae strain DBVPG1373, a soil isolate from the Netherlands, with S. paradoxus

76 strain Z1, an English oak tree isolate. The resulting sterile hybrid had a thermotolerance

77 phenotype between those of its purebred parents (Figure S4). In this hybrid background we

78 generated hemizygote mutants using a plasmid-borne, selectable PiggyBac transposon

79 system ${ }^{19}$. We cultured the pool of mutants in bulk for $\sim 7$ generations at $39^{\circ} \mathrm{C}$ and, separately, at

$8028^{\circ} \mathrm{C}$. From cells in each culture we sequenced transposon insertion locations ${ }^{20}$ as a readout of

81 the genotypes and abundance of mutant hemizygote clones present in the selected sample. In

82 these sequencing data, at each of 4888 genes we detected transposon mutant clones in both 
83 species' alleles in the hybrid (Figure S5), with transposon insertions distributed in a largely

84 unbiased manner across the genome (Figure S6). For a given gene, we tabulated the

85 abundances of mutants whose transposon insertion fell in the S. cerevisiae allele of the hybrid, 86 after high-temperature selection relative to the $28^{\circ} \mathrm{C}$ control, and we compared them to the

87 abundance distribution of mutants in the $S$. paradoxus allele (Figure 2A). Any difference in 88 abundance between these reciprocal hemizygote cohorts can be ascribed to variants between 89 the retained alleles at the respective locus; we refer to the comparison as reciprocal 90 hemizygosity analysis via sequencing ( $\mathrm{RH}$-seq). Integrating this approach with a quality-control 91 pipeline (Figure S5), in a survey of 3416 high-coverage genes we identified 8 top-scoring hits

92 (false discovery rate 0.01 ; Figure 2B). At each such locus, disruption of the $S$. cerevisiae allele

93 in the hybrid was associated with low clone abundance after selection at $39^{\circ} \mathrm{C}$ relative to $28^{\circ} \mathrm{C}$

94 (Figure 2B), reflecting a requirement for the S. cerevisiae allele for thermotolerance. None of the 95 genes mapped by $\mathrm{RH}$-seq had a known role in the yeast heat shock or stress responses. All 96 were annotated as housekeeping factors: ESP1, DYN1, MYO1, CEP3, APC1, and SCC2

97 function in chromosome segregation and cytokinesis, and AFG2 and TAF2 in

98 transcription/translation.

100 To evaluate the role in thermotolerance of genes that emerged from $\mathrm{RH}$-seq, we first sought to 101 verify that growth differences between hemizygotes at a given locus were the consequence of 102 allelic variation and not an artifact of our genomic approach. As a complement to our analysis of 103 transposon mutants, for each $\mathrm{RH}$-seq hit gene we engineered hemizygotes by targeted deletion 104 of each species' allele in turn in the hybrid. In growth assays, the strain lacking the S. cerevisiae 105 allele at each gene grew poorly at high temperature (Figure 2B), with little impact at $28^{\circ} \mathrm{C}$ 106 (Figure S7), consistent with fitness inferences from $\mathrm{RH}$-seq. And in each case, the S. paradoxus 107 allele made no contribution to the phenotype of the hybrid, since deleting it had no effect 
108 (Figures 2B and S7). We conclude that the loci emerging from $\mathrm{RH}$-seq represent bona fide

109 determinants of thermotolerance in the hybrid.

111 Validation of RH-seq gene hits in purebreds

113 We expected that variation at our $\mathrm{RH}$-seq hits, though mapped by virtue of their impact in the

114 hybrid, could also explain thermotolerance differences between purebred species. As a test of

115 this notion, for each mapped gene in turn, we replaced the two copies of the endogenous allele

116 in each purebred diploid with the allele from the other species. Growth assays of these

117 transgenics established the $S$. cerevisiae allele of each locus as necessary or sufficient for

118 biomass accumulation at $39^{\circ} \mathrm{C}$, or both: thermotolerance in the $S$. cerevisiae background was

119 compromised by $S$. paradoxus alleles at 7 of the 8 genes and, in S. paradoxus, improved by $S$.

120 cerevisiae alleles at 6 of 8 loci (Figure 3). Allele replacements had little impact on growth at

$12128^{\circ} \mathrm{C}$ (Figure S8). These trends mirrored the direction of locus effects from hemizygotes in the

122 hybrid, though the magnitudes were often different. Most salient were the small effect sizes in S.

123 paradoxus relative to other backgrounds, indicative of strong epistasis in this poorly-performing

124 species (Figure S9). Thus, the loci mapped by $\mathrm{RH}$-seq in an interspecies hybrid contribute

125 causally to thermotolerance in purebreds, with effect sizes that depend on the context in which

126 they are interrogated.

128 Avid growth at high temperature is a defining characteristic of $S$. cerevisiae as a species, 129 relative to other Saccharomycetes (refs. 14-16 and Figure 1). In principle, the loci mapped by $130 \mathrm{RH}-$ seq could be unique to the genetic architecture of thermotolerance in our focal S. cerevisiae 131 strain, DBVPG1373, or be part of a mechanism common to many S. cerevisiae isolates. In 132 support of the latter model, transgenesis experiments showed that a diverse panel of $S$. 133 cerevisiae isolates all harbored alleles conferring modest but significant growth benefits at high 
134 temperature, and alleles from multiple S. paradoxus isolates were deleterious (Figure S10A-B).

135 We detected no such impact at $28^{\circ} \mathrm{C}$ (Figure S10A-B). Similarly, we found elevated sequence

136 divergence from S. paradoxus to be a shared feature of S. cerevisiae strains at the loci mapped

137 by $\mathrm{RH}$-seq (using the absolute divergence measure $\mathrm{D}_{\mathrm{xy}}$; Figure $\mathrm{S} 10 \mathrm{C}$ ). These findings indicate

138 that the S. cerevisiae population accumulated divergent, pro-thermotolerance alleles at the loci

139 mapped by $\mathrm{RH}$-seq, consistent with a role in the trait for these genes across the species.

140 Likewise, in the yeast phylogeny, $\mathrm{RH}$-seq hit genes were distinguished by accelerated evolution

141 along the branch leading to $S$. cerevisiae, as expected if the ancestral program has been

142 conserved among the other species in the clade (Figure S10C).

144 Discussion

146 In this work, we have developed the $\mathrm{RH}$-seq method for genome-wide mapping of natural trait

147 variation, and we have used it to elucidate the genetics of thermotolerance in reproductively 148 isolated yeasts. Growth at high temperature is likely a derived character in S. cerevisiae ${ }^{14-16}$, 149 and the mechanism by which evolution built the trait, after the split from S. paradoxus, has 150 remained unknown. In pursuing the genetics of this putative ancient adaptation, we complement 151 studies of younger, intra-specific variants that erode thermotolerance, in the few S. cerevisiae 152 strains that have lost the trait relatively recently ${ }^{12,21}$. We have sought to shed light on more 153 ancient evolutionary events by considering S. paradoxus as a representative of the ancestral 154 state, to which thermotolerant S. cerevisiae can be compared.

156 Using this approach, we have mapped eight loci at which S. cerevisiae alleles are necessary 157 and sufficient for thermotolerance. Six of these genes are essential for growth in standard 158 conditions $^{22}$, and all eight contribute to fundamental growth processes. ESP1, DYN1, CEP3, 
159 APC1, MYO1, and SCC2 mediate mitotic spindle assembly, chromatid cohesion and separation,

160 cytokinesis, and mitotic exit; AFG2 regulates the release of maturation factors from the

161 ribosome; and TAF2 encodes a TFIID subunit. In each case, our growth experiments in the

162 interspecific hybrid have shown that the S. paradoxus allele acts as a hypomorph at high

163 temperature. Our work leaves unanswered exactly how heat-treated S. paradoxus cells die in

164 the absence of these functions, though their large-budded morphology strongly suggests

165 regulated arrest or stochastic failure late in the cell cycle. Given that some but not all $\mathrm{RH}$-seq hit

166 loci have roles in mitosis, it is likely only one of the choke points at which S. paradoxus alleles

167 are a liability at high temperature. Assuming that these heat-sensitive alleles also littered the

168 genome of the common ancestor with S. cerevisiae, thermotolerance would have evolved along

169 the S. cerevisiae lineage by resolving each of them, boosting the heat resistance of many

170 housekeeping processes. Such a mechanism would dovetail with the recent finding that, across

171 species, the limiting temperature for cell growth correlates with the melting temperatures of a

172 subset of essential proteins ${ }^{23}$.

174 These insights into the evolution of a complex yeast trait serve as a proof of concept for $\mathrm{RH}-$

175 seq. To date, the reciprocal hemizygosity test has led to landmark discoveries in a candidate-

176 gene framework, confirming the effects of variation at a given locus identified by other

177 means ${ }^{12,13}$. Schemes to scale up the test have generated a genome's worth of hemizygotes

178 from deletion-strain purebreds, which tend to harbor secondary mutations that come through

179 screens as false positives ${ }^{24,25}$. As such, a key advantage of $\mathrm{RH}$-seq is that we carry out

180 mutagenesis in the hybrid, which ensures coverage of essential genes and obviates the use of

181 mutation-prone null genotypes. Furthermore, any secondary mutations that do arise in a given

182 hemizygote clone should have little influence in $\mathrm{RH}$-seq mapping, because deep mutagenesis

183 generates many independent clones per gene that are analyzed together. One important caveat

184 of $\mathrm{RH}$-seq, as in single-gene reciprocal hemizygote tests, is the assumption that no epistasis 
185 unique to the hybrid will mask the effects of loci underlying a trait difference of interest between

186 the parents. In our case study, the genetic architecture of thermotolerance in the hybrid did bear

187 out as relevant for the purebreds, albeit with locus effect sizes that varied across the

188 backgrounds. More dramatic discrepancies may be particularly likely when the hybrid has a

189 heterotic (i.e. extreme) phenotype and is a poor model for the genetics of the parents ${ }^{26}$. The

190 choice of a non-heterotic hybrid in which to pursue $\mathrm{RH}$-seq would be analogous to classical

191 linkage mapping in a cross whose progeny have, on average, phenotypes that are intermediate

192 between those of the parents.

193

194 In fact, although we have focused here on ancient divergence, the RH-seq method would be

195 just as applicable to individuals within a species, as a high-resolution alternative to linkage

196 analysis. We thus anticipate that $\mathrm{RH}$-seq will accelerate the mapping of genotype to phenotype

197 in many systems, whether the parents of a cross are closely related or members of a species

198 complex that have been locally adapting for millions of years. 

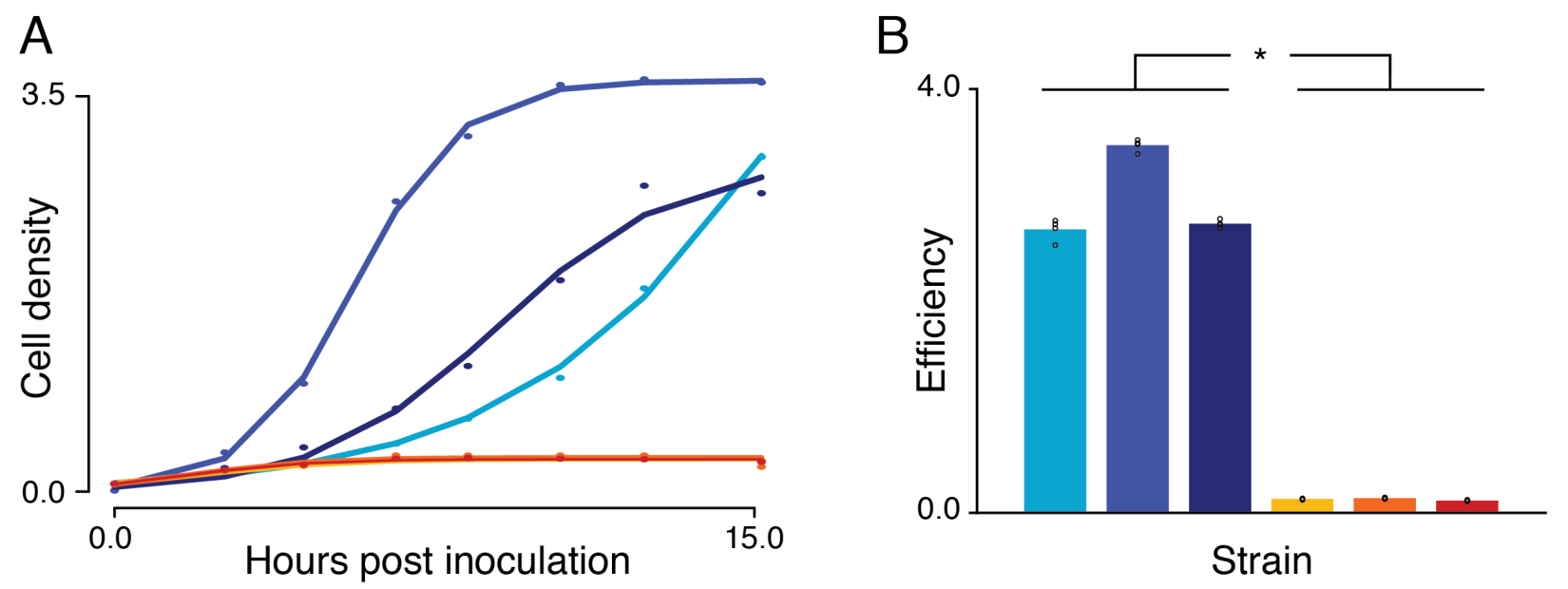

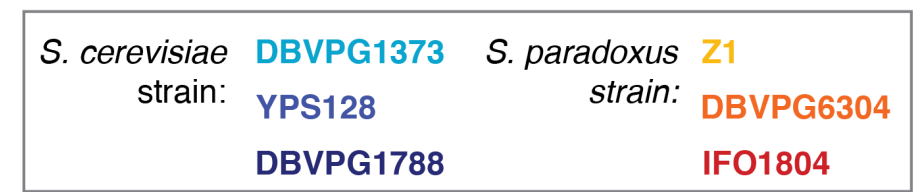

202 Figure 1. S. cerevisiae grows better at high temperature than S. paradoxus.

203 A, Each point reports cell density $\left(\mathrm{OD}_{600}\right)$ of the indicated wild isolate of S. cerevisiae (blue) or

204 S. paradoxus (orange) over a timecourse of growth at $39^{\circ} \mathrm{C}$. Each solid line shows a logistic 205 population growth model fit to the respective cell density measurements. B, Each bar reports 206 mean efficiency $(n=4)$ of the indicated strain at $39^{\circ} \mathrm{C}$, defined as the difference between cell

207 density at 24 hours of growth and that at the time of inoculation. *, $p=3.78 \times 10^{-11}$; individual 208 measurements are reported as open circles. 
213 Figure 2

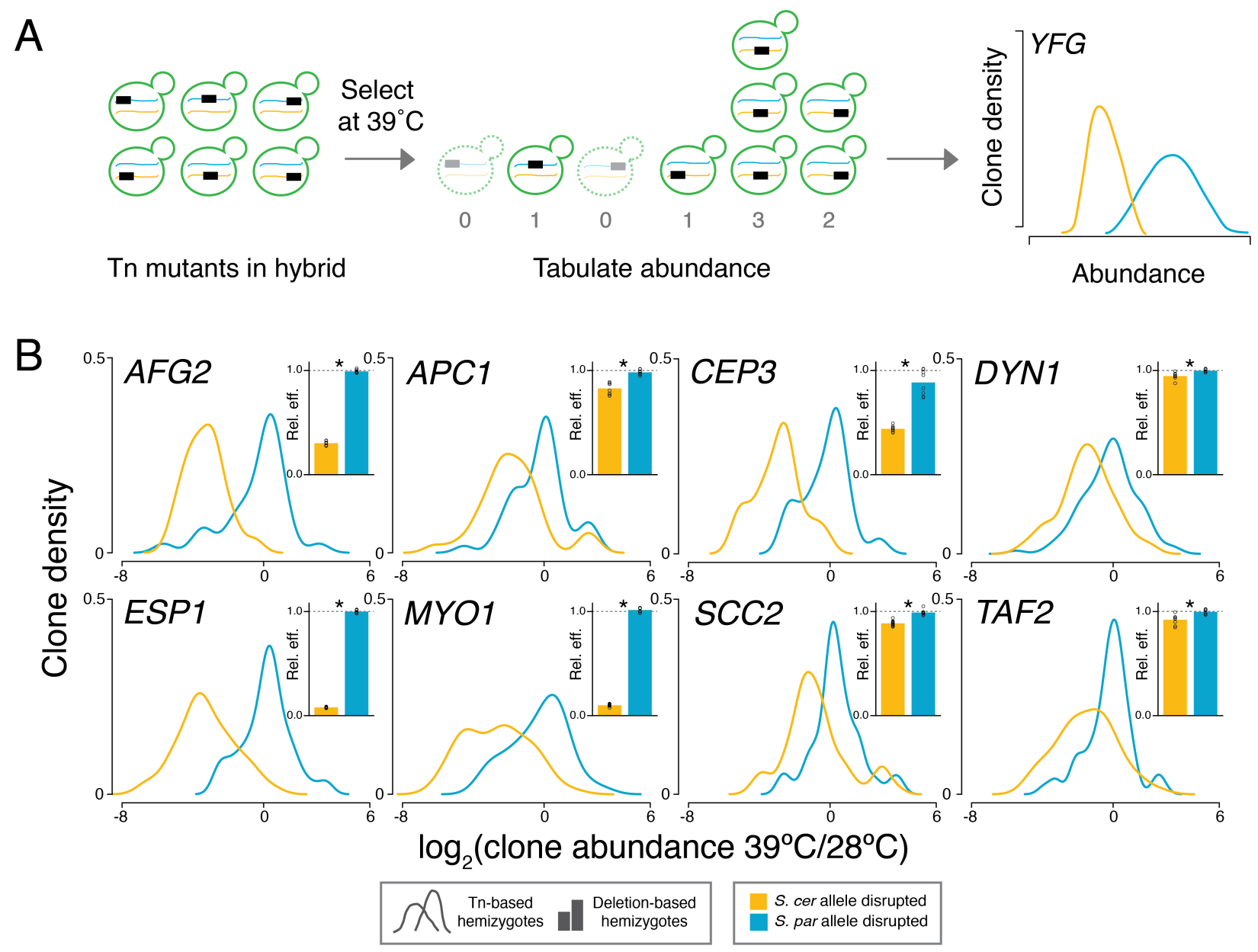

Figure 2. Mapping thermotolerance by RH-seq.

216 A, A transposon (rectangle) disrupts the allele from S. cerevisiae (blue) or S. paradoxus

217 (orange) of a gene (YFG) in an interspecific hybrid (green). Clones lacking the pro-

218 thermotolerance allele grow poorly at $39^{\circ} \mathrm{C}$ (dashed outlines), as measured by sequencing and

219 reported in smoothed histograms (traces, colored to indicate the species' allele that is not

220 disrupted). B, Each panel reports results from one $\mathrm{RH}$-seq hit locus. In the main figure of a

221 given panel, the $x$-axis reports the $\log _{2}$ of abundance, measured by $\mathrm{RH}$-seq after selection at

$22239^{\circ} \mathrm{C}$, of a clone harboring a transposon insertion in the indicated species' allele, relative to the

223 analogous quantity for that clone from selection at $28^{\circ} \mathrm{C}$. The $y$-axis reports the proportion of all

224 clones bearing insertions in the indicated allele that exhibited the abundance ratio on the $x$, as a 
225 kernel density estimate. In insets, each bar reports the relative efficiency, calculated as the

226 mean growth efficiency at $39^{\circ} \mathrm{C}(n=8-12)$ of the indicated targeted-deletion hemizygote

227 measured in liquid culture assays, normalized to the analogous quantity for the wild-type hybrid.

$228 *, p \leq 0.002$; individual measurements are reported as open circles. 
A
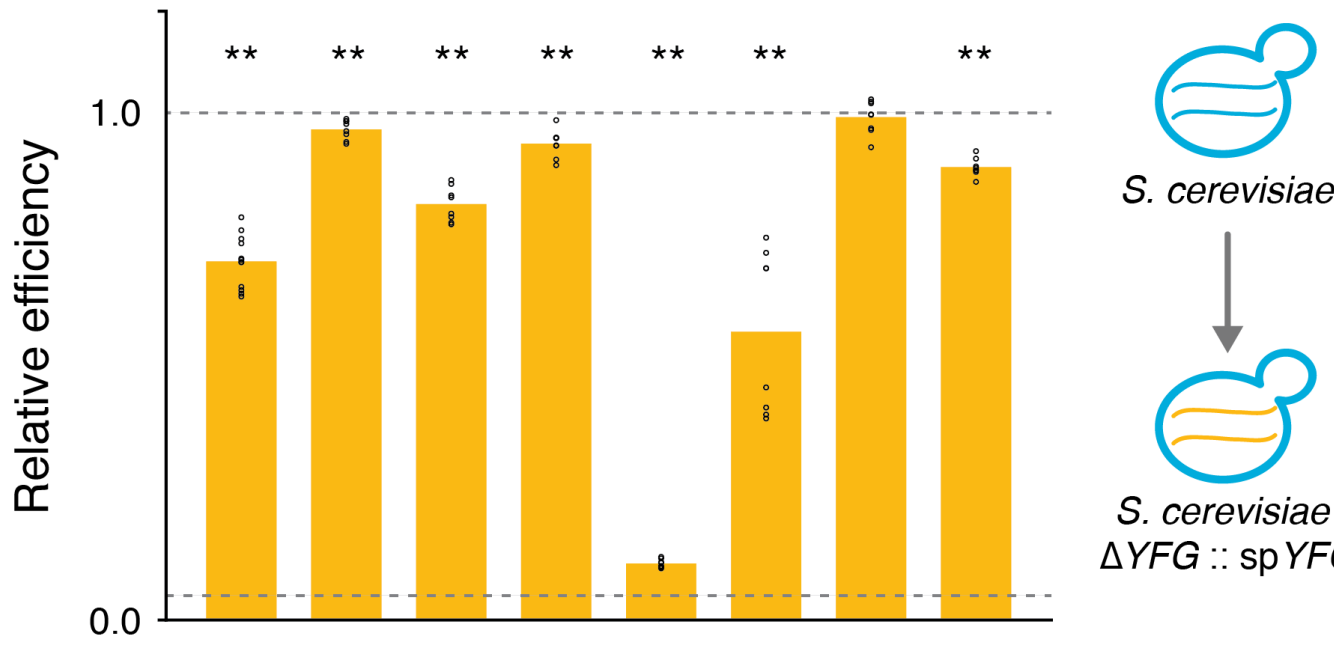

S. cerevisiae

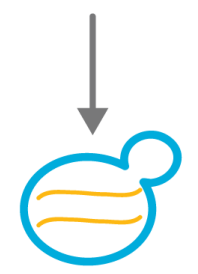

S. cerevisiae $\triangle Y F G:: \mathrm{sp} Y F G$

B
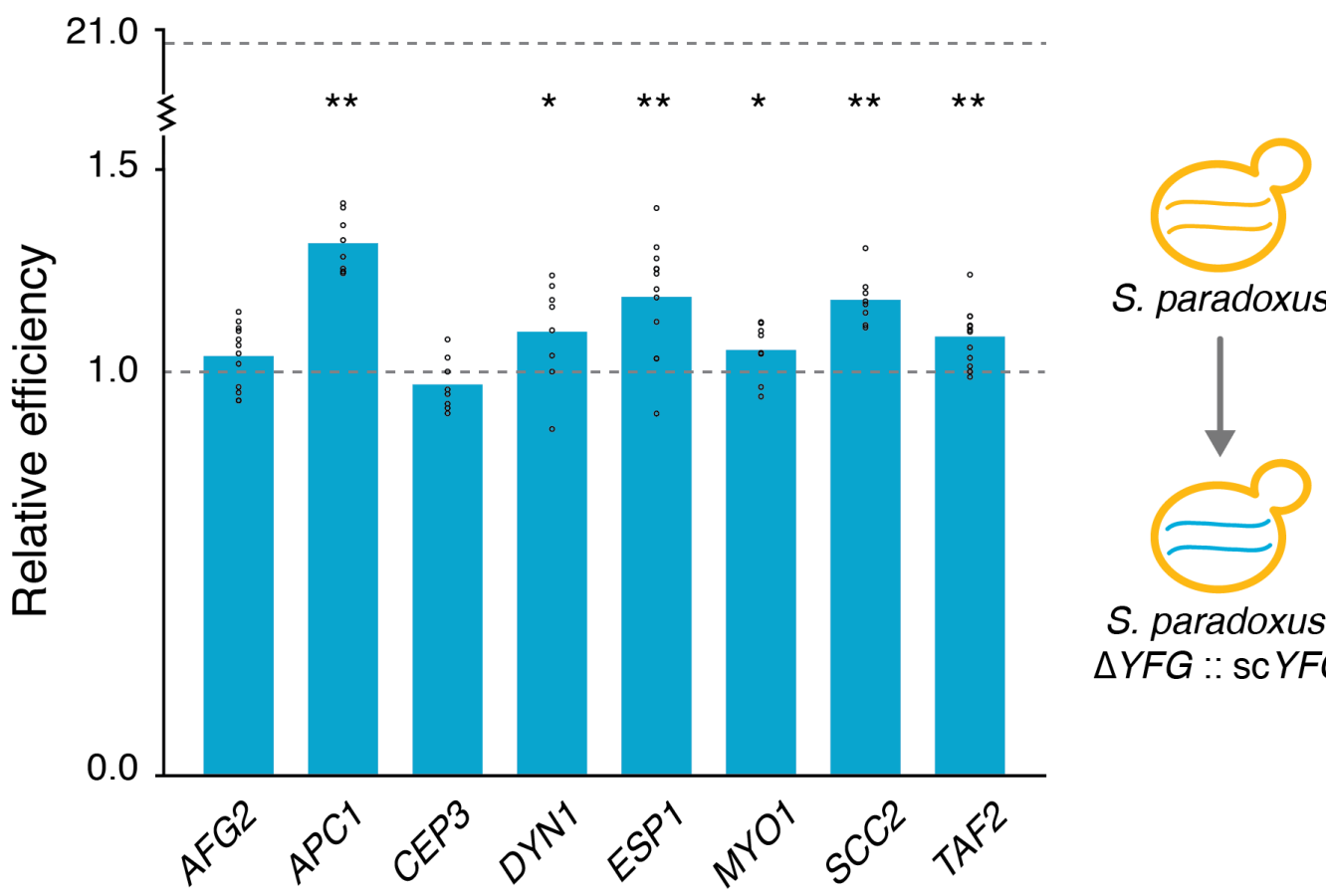

S. paradoxus

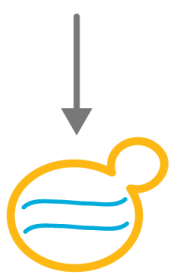

S. paradoxus

$\triangle Y F G::$ sc $Y F G$

\section{Figure 3. S. cerevisiae thermotolerance alleles are necessary and sufficient for growth at}

\section{3 high temperature.}

234 A, Each bar reports mean growth efficiency at $39^{\circ} \mathrm{C}$, measured in liquid culture assays $(n=6$ -

235 12), of an S. cerevisiae strain harboring the S. paradoxus allele at the indicated RH-seq hit

236 locus, relative to the analogous quantity for wild-type S. cerevisiae. B, Data are as in A, except 
237 that each bar reports results from a S. paradoxus strain harboring the S. cerevisiae allele at the

238 indicated locus, normalized to wild-type S. paradoxus. In a given panel, the top and bottom

239 dotted lines report the relative efficiency of wild-type S. cerevisiae and S. paradoxus,

240 respectively. ${ }^{*}, p \leq 0.036 ;{ }^{* *}, p \leq 0.001$. Individual measurements are reported as open circles. 


\section{Materials and Methods}

\section{Strains}

246 Strains used in this study are listed in Table S1. Homozygous diploid strains of S. cerevisiae

247 and S. paradoxus used as parents of the interspecific hybrid, and as the backgrounds for allele-

248 swap experiments, were homothallic DBVPG1373 and Z1, respectively. In the case of the

249 hybrid parents, each strain was rendered homozgyous null for URA3 via homologous

250 recombination with a HYGMX cassette, then sporulated; a given mated spore from a dissected

251 tetrad was grown up into a diploid that was homozygous null at URA3 and tested for the

252 presence of both genomes by PCR with species-specific primers.

\section{4 piggyBac transposon machinery}

256 For untargeted, genome-scale construction of reciprocal hemizygotes in the S. cerevisiae $\times$ S.

257 paradoxus hybrid, we adapted methods for piggyBac transposon mutagenesis ${ }^{19}$ to develop a

258 system in which the transposon machinery was borne on a selectable and counter-selectable

259 plasmid lacking a centromere. We constructed this plasmid (final identifier pJR487) in three

260 steps. In step I we cloned the piggyBac transposase enzyme gene driven by the S. cerevisiae

$261 T D H 3$ promoter (from plasmid p3E1.2, a gift from Malcolm Fraser, Notre Dame) into plasmid

262 pJED104 (which contains URA3, an ARS, and the CEN6 locus, and was a gift from John

263 Dueber, UC Berkeley). For this cloning, the amplification used a forward and reverse primer

264 containing a BamHI and Xhol site, respectively, that upon restriction digest yielded sticky ends

265 for ligation to recipient BamHI and Xhol sites in digested pJED104. We used the resulting

266 plasmid as input into step II, removal of the CEN6 sequence: we first amplified the entire 
267 plasmid with primers that initiated outside of CEN6 and were directed away from it, and

268 contained reciprocally complementary Nhel sites; sticky ends of the linear PCR product were

269 then ligated together for re-circularization. We used the resulting plasmid as input into step III,

270 the cloning in of a construct comprised of the KANMX cassette flanked by long terminal arms

271 (328bp and 361bp) from the piggyBac transposon. We first amplified KANMX from pUG6 $6^{27}$ and

272 each transposon arm from p3E1.2, using primers that contained overlapping sequence on the

273 fragment ends that would ultimately be the interior of the construct, and Xbal sites on the

274 fragment ends that would ultimately be the 5' and 3'-most ends of the construct. We stitched the

275 three fragments together by overlap extension PCR, digested the resulting construct and the

276 plasmid from step II with Xbal, and annealed sticky ends of the two to yield the final pJR487

277 plasmid.

Untargeted hemizygote construction via transposon mutagenesis

281 For mutagenesis, pJR487 was gigaprepped using a column kit (Zymo Research) to generate

$282 \sim 11 \mathrm{mg}$ plasmid. To prepare for transformation, JR507 (the S. cerevisiae DBVPG1373 x S.

283 paradoxus $\mathrm{Z} 1$ hybrid) was streaked from a $-80^{\circ} \mathrm{C}$ freezer stock onto a yeast peptone dextrose

284 (YPD, 1\% yeast extract [BD], 2\% yeast peptone [BD], 2\% D-glucose [Sigma]) agar plate and

285 incubated for 2 days at $26^{\circ} \mathrm{C}$. A single colony was inoculated into $100 \mathrm{~mL}$ YPD and shaken at

$28628^{\circ} \mathrm{C}, 200 \mathrm{rpm}$ for $\sim 24$ hours. The next day, we transferred cells from this pre-culture, and YPD,

287 to each of four $1 \mathrm{~L}$ flasks at the volumes required to attain an optical density at $600 \mathrm{~nm}\left(\mathrm{OD}_{600}\right)$

288 of 0.2 in $500 \mathrm{~mL}$ each. We cultured each for 6 hours at $28^{\circ} \mathrm{C}$ with shaking at $200 \mathrm{rpm}$. Two of

289 these cultures were combined into $1 \mathrm{~L}$ of culture and two into a separate $1 \mathrm{~L}$, and each such

290 culture was subjected to transformation (for a total of two transformations) as follows. The $1 \mathrm{~L}$

291 was split into twenty 50-mL conical tubes. Each aliquot was centrifuged and washed with water

292 and then with $0.1 \mathrm{M}$ lithium acetate (LiOAc, Sigma) mixed with 1X Tris-EDTA buffer (10 mM 
293 Tris- $\mathrm{HCl}$ and $1.0 \mathrm{mM}$ EDTA); after spin-down, to each tube was added a solution of $0.269 \mathrm{mg}$ of

294 pJR487 mixed 5:1 by volume with salmon sperm DNA (Invitrogen), and then to each was added

$2953 \mathrm{~mL}$ of $39.52 \%$ polyethylene glycol, 0.12M LiOAc and 1.2X Tris-EDTA buffer (12 mM Tris- $\mathrm{HCl}$

296 and $1.2 \mathrm{mM}$ EDTA). Tubes were rested for 10 minutes at room temperature, then heat-shocked

297 in a water bath at $39^{\circ} \mathrm{C}$ for 26 minutes. Cells from all 20 tubes were then combined. We

298 transferred cells from this post-transformation culture, and YPD, to each of three $1 \mathrm{~L}$ flasks at

299 the volumes required to attain an $\mathrm{OD}_{600}$ of $\sim 0.35-4$ in $500 \mathrm{~mL}$. Each such culture was recovered

300 by shaking at $28^{\circ} \mathrm{C}$ and $200 \mathrm{rpm}$ for 2 hours. G418 (Geneticin, Gibco) was added to each at a

301 concentration of $300 \mu \mathrm{g} / \mathrm{mL}$ to select for those cells which had taken up the plasmid, and

302 cultures were incubated with $200 \mathrm{rpm}$ shaking at $28^{\circ} \mathrm{C}$ for two days until each reached an $\mathrm{OD}_{600}$

303 of $\sim 2.3$. All six such selected cultures across the two transformations were combined. We

304 transferred cells from this combined culture, and YPD + G418 $(300 \mathrm{ug} / \mathrm{mL})$, to each of two $1 \mathrm{~L}$

305 flasks at the volumes required to attain an $\mathrm{OD}_{600}$ of 0.2 in $500 \mathrm{~mL}$ each. We cultured each flask

306 at $28^{\circ} \mathrm{C}$ and $200 \mathrm{rpm}$ shaking overnight until reaching an $\mathrm{OD}_{600}$ of 2.18 and combined the two

307 cultures again to yield one culture. To cure transformants of the pJR487 URA+ plasmid, we

308 spun down a volume of this master culture and resuspended in water with the volume required

309 to attain a cell density of $1.85 \mathrm{OD}_{600}$ units $/ \mathrm{mL} .12 \mathrm{~mL}$ of this resuspension were plated $(1 \mathrm{~mL}$ per

$31024.1 \mathrm{~cm} \times 24.1 \mathrm{~cm}$ plate) onto plates containing complete synthetic media with 5-fluoroorotic acid

311 (5-FOA) [0.2\% drop-out amino acid mix without uracil or yeast nitrogen base (YNB) (US

312 Biological), 0.005\% uracil (Sigma), 2\% D-glucose (Sigma), 0.67\% YNB without amino acids

313 (Difco), $0.075 \%$ 5-FOA (Zymo Research)]. After incubation at $28^{\circ} \mathrm{C}$ to enable colony growth,

314 colonies were scraped off all 12 plates and combined into water at the volume required to attain

$31540 \mathrm{OD}_{600}$ units per $900 \mu \mathrm{L}$, yielding the final transposon mutant hemizygote pool. This was

316 aliquoted into $1 \mathrm{~mL}$ volumes with $10 \% \mathrm{DMSO}$ and frozen at $-80^{\circ} \mathrm{C}$. 
320 One aliquot of the pool of transposon mutant hemizygotes in the JR507 S. cerevisiae

321 DBVPG1373 x S. paradoxus Z1 hybrid background was thawed and inoculated into $150 \mathrm{~mL}$ of

322 YPD in a $250 \mathrm{~mL}$ flask, and cultured for 7.25 hours at $28^{\circ} \mathrm{C}$, with shaking at $200 \mathrm{rpm}$. We used

323 this timepoint as time zero of our thermotolerance experiment, and took four aliquots of $6.43 \mathrm{~mL}$

324 (7 OD units) as technical replicates for sequencing of transposon insertion positions (see

325 below). $9.19 \mathrm{~mL}$ of the remaining culture was back-diluted to an $\mathrm{OD}_{600}$ of 0.02 in a total of 500

$326 \mathrm{~mL}$ YPD in each of six $2 \mathrm{~L}$ glass flasks for cultures that we call selections; three were grown at

$32728^{\circ} \mathrm{C}$ and three at $39^{\circ} \mathrm{C}$ (shaking at $200 \mathrm{rpm}$ ) until an $\mathrm{OD}_{600}$ of $1.9-2.12$ was reached,

328 corresponding to about 6.5 doublings in each case. Four cell pellets of $7 \mathrm{OD}_{600}$ units each were

329 harvested from each of these biological replicate flasks, for sequencing as technical replicates

330 (see below). In total, 28 pellets were subjected to sequencing: 4 technical replicates from the

331 time-zero culture; 3 biological replicates, 4 technical replicates each, from the $28^{\circ} \mathrm{C}$ selection;

332 and 3 biological replicates, 4 technical replicates each, from the $39^{\circ} \mathrm{C}$ selection.

\section{Tn-seq library construction}

336 To determine the abundance of transposon mutant hemizygote clones after selection, we first

337 sequenced transposon ( $\mathrm{Tn}$ ) insertions as follows. Each cell pellet from a time zero or selection

338 sample (see above) was thawed on ice, and its genomic DNA (gDNA) was harvested with the

339 ZR Fungal/Bacterial DNA MiniPrep kit (Zymo Research). gDNA was resuspended in DNA

340 elution buffer (Zymo) pre-warmed to $65^{\circ} \mathrm{C}$ and its concentration was quantified using a Qubit 3.0

341 fluorometer. Illumina Tn-seq library construction was as described ${ }^{28}$. Briefly, gDNA was

342 sonicated and ligated with common adapters, and for each fragment deriving from a Tn insertion

343 in the genome, a sequence containing a portion of the transposon and a portion of its genomic

344 context (the Tn-genome junction) was amplified using one primer homologous to a region in the 
345 transposon, and another primer homologous to a region in the adapter. The indexed adapter-

346 specific primer was

347 CAAGCAGAAGACGGCATACGAGATNNNNNNGTGACTGGAGTTCAGACGTGTGCTCTTCCG

348 ATCT, where the six N's are a unique index used for multiplexing multiple libraries onto the

349 same Hiseq sequencing lane, and the transposon specific primer was

350 ATGATACGGCGACCACCGAGATCTACACTCTTTCCCTACACGACGCTCTTCCGATCT

351 NNNNNNAGCAATATTTCAAGAATGCATGCGTCAAT, where N's are random nucleotides.

352 Amplification used Jumpstart polymerase (Sigma) and the following cycling protocol: $94^{\circ} \mathrm{C}-2$

$353 \mathrm{~min},\left[94^{\circ} \mathrm{C}-30 \mathrm{sec}, 65^{\circ} \mathrm{C}-20 \mathrm{sec}, 72^{\circ} \mathrm{C}-30 \mathrm{sec}\right] \times 25,72^{\circ} \mathrm{C}-10 \mathrm{~min}$. Sequencing of single-end

354 reads of $150 \mathrm{bp}$ was done over eight lanes on a HiSeq 2500 at the Joint Genome Institute

355 (Walnut Creek, CA). Reads sequenced per library are reported in Table S2.

356

357 Tn-seq read-mapping and data analysis

359 For analysis of data from the sequencing of Tn insertion sites in pools of hemizygotes, we first

360 searched each read for a string corresponding to the last 20 base pairs of the left arm of the

361 piggyBac transposon sequence, allowing up to two mismatches. For each Tn-containing read,

362 we then identified the genomic location of the sequence immediately downstream of the $\mathrm{Tn}$

363 insertion site, which we call the genomic context of the insertion, by mapping with BLAT

364 (minimum sequence identity $=95$, tile size $=12$ ) against a hybrid reference genome made by

365 concatenating amended S. cerevisiae DBVPG1373 and S. paradoxus Z1 genomes (see below).

366 These genomic-context sequence fragments were of variable length; any case in which the

367 sequence was shorter than 50 base pairs was eliminated from further analysis, as was any case

368 in which a genomic-context sequence mapped to more than one location in the hybrid

369 reference. The resulting data set thus comprised reads containing genomic-context sequences

370 specifically mapping to a single location in either S. cerevisiae DBVPG1373 or S. paradoxus Z1, 
371 which we call usable reads. For a given library, given a cohort of usable reads whose genomic-

372 context sequence mapped to the same genomic location, we inferred that these reads

373 originated from clones of a single mutant with the Tn inserted the respective site, which we

374 call an insertion. In cases where the genomic-context sequences from reads in a given library

375 mapped to positions within 3 bases of each other, we inferred that these all originated from the

376 same mutant genotype and combined them, assigning to them the position corresponding to the

377 single location to which the most reads mapped among those combined. For a given insertion

378 thus defined, we considered the number of associated reads $n_{\text {insert }}$ as a measure proportional to

379 the abundance of the insertion clone in the cell pellet whose gDNA was sequenced. To enable

380 comparison of these abundances across samples, we tabulated the total number of usable

381 reads $n_{\text {pellet }}$ from each cell pellet, took the average of this quantity across pellets, $\left\langle n_{\text {pellet }}\right\rangle$, and

382 multiplied each $n_{\text {insert }}$ by $<n_{\text {pellet }}>/ n_{\text {pellet }}$ to yield $a_{\text {insert }}$, the final normalized estimate of the

383 abundance of the insertion clone in the respective pellet. For any insertions that were not

384 detected in a given pellet's library $\left(n_{\text {insert }}=0\right)$ but detectable in another library of the data set, we

385 assigned $n_{\text {insert }}=1$.

386

387 We evaluated, from the mapped genomic-context sequence of each insertion, whether it fell into

388 a gene according to the S. cerevisiae and S. paradoxus genome annotations ${ }^{17,29}$, and we

389 retained for further analysis only those insertions that fell into genes that were syntenic in the

390 two species. For each such insertion, for each biological replicate corresponding to a selection

391 culture (at $28^{\circ} \mathrm{C}$ or $39^{\circ} \mathrm{C}$ ), we averaged the normalized abundances $a_{\text {insert }}$ across technical

392 replicates, yielding a single abundance estimate $<a_{\text {insert }}>_{\text {technical }}$ for the biological replicate. We

393 then calculated the mean of the latter quantities across all biological replicates of the selection,

394 to yield a final abundance estimate for the insertion in this selection, $\left.<a_{\text {insert }}\right\rangle_{\text {total }}$. Likewise, for

395 each insertion and selection experiment we calculated $C V_{\text {insert, total, the coefficient of variation of }}$

$396<a_{\text {insert }}>_{\text {technical }}$ values across biological replicates. 
398 To use Tn-seq data in reciprocal hemizygosity tests, we considered for analysis only genes

399 annotated with the same (orthologous) gene name in the S. cerevisiae and S. paradoxus

400 reference genomes. For each insertion, we divided the $\left.<\mathrm{a}_{\text {insert }}\right\rangle_{\text {total }}$ value from the $39^{\circ} \mathrm{C}$ selection

401 by the analogous quantity from the $28^{\circ} \mathrm{C}$ selection and took the $\log _{2}$ of this ratio, which we

402 consider to reflect thermotolerance as measured by $\mathrm{RH}$-seq. For each gene in turn, we used a

403 two-tailed Mann-Whitney $U$ test to compare thermotolerance measured by $\mathrm{RH}$-seq between the

404 set of insertions falling into the $S$. cerevisiae alleles of the gene, against the analogous quantity

405 from the set of insertions falling into the $S$. paradoxus allele of the gene, and we corrected for

406 multiple testing using the Benjamini-Hochberg method.

408 We tabulated the number of inserts and genes used as input into the reciprocal hemizygote test,

409 and the number of top-scoring genes emerging from these tests, under each of a range of

410 possible thresholds for coverage and measurement noise parameter values (Figure S5). We

411 used in the final analysis the parameter-value set yielding the most extensive coverage and the

412 most high-significance hits: this corresponded to insertions whose abundances had, in the data

413 from at least one of the two selections (at $28^{\circ} \mathrm{C}$ or $39^{\circ} \mathrm{C}$ ), $C V_{\text {insert, total }} \leq 1.5$ and $\left.<a_{\text {insert }}\right\rangle_{\text {total }} \geq 1.1$,

414 and genes for which this high-confidence insertion data set contained at least 5 insertions in

415 each species' allele. This final data set comprised 110,678 high-quality insertions (Table S3) in

4163416 genes (Table S4).

418 Amended reference genome construction

420 We generated reference genomes for S. cerevisiae strain DBVPG1373 and S. paradoxus strain

421 Z1 as follows. Raw genome sequencing reads for each strain were downloaded from the

422 SGRP2 database (ftp://ftp.sanger.ac.uk/pub/users/dmc/yeast/SGRP2/input/strains). Reads were 
423 aligned using bowtie $2^{30}$ with default options; DBVPG1373 reads were aligned to version

424 R64.2.1 of the reference sequence of the S. cerevisiae type strain S288C (Genbank Assembly

425 Accession GCA_000146045.2), and Z1 reads were aligned to the S. paradoxus strain CBS432

426 reference sequence ${ }^{31}$. Single nucleotide variants (SNPs) were called using a pipeline of

427 samtools ${ }^{32}$, bcftools and bgzip, and were filtered for a quality score (QUAL) of $>20$ and a

428 combined depth (DP) of $>5$ and either $<65$ (S. cerevisiae) or $<255$ (S. paradoxus). We then

429 amended each reference genome with the respective filtered SNPs: we replaced the S288C

430 allele with that of DBVPG1373 at each filtered SNP using bcftools' consensus command with

431 default options (42,983 base pairs total), and amendment of the CBS432 sequence was carried

432 out analogously using Z1 alleles (15,126 base pairs total).

\section{Targeted-deletion hemizygote construction by homologous recombination}

436 A given targeted hemizygote for each $\mathrm{RH}$-seq hit gene except TAF2 was generated in the $\mathrm{S}$.

437 cerevisiae DBVPG1373 x S. paradoxus Z1 hybrid (JR507) by knocking out the allele of the gene 438 from one species via homologous recombination with KANMX as described ${ }^{33}$ with 70 base pairs 439 of homology on the 5' and 3' ends of the cassette; checking was via diagnostic PCR. Two or 440 more independent transformants were isolated and phenotyped for each hemizygote genotype 441 (Table S1).

\section{Construction of allele replacement and targeted hemizgyote strains with Cas9}

445 At each $\mathrm{RH}$-seq hit gene, we constructed strains in wild-type homozygous diploid S. cerevisiae 446 DBVPG1373 in which both copies of the endogenous allele were replaced by the allele from an

447 S. paradoxus isolate (Z1 for Figures 3, S8, and S9, and other strains as indicated for Figure 448 S10), and likewise for replacement of alleles from S. cerevisiae (DBVPG1373 in Figures 3, S8, 
449 and S9, and other strains as indicated for Figure S10) into S. paradoxus Z1. We call each such

450 strain an allele-replacement strain, and each was constructed using a dual-guide Cas9

451 transgenesis method ${ }^{34}$ in which a linear PCR fragment from the donor species is incorporated

452 into the recipient genome by homology-directed repair of two chromosomal double-strand

453 breaks induced by Cas9. Briefly, for each allele of each gene, we designed two guide RNAs for

454 double-strand breaks by Cas9: one guide targeted a position $\sim 1000$ base pairs 5 ' to the coding

455 start or at the 3' end of the closest upstream gene, whichever was closer, and the other guide

456 targeted the region of the coding stop. The precise cut site of each was chosen to contain an

457 NGG immediately downstream of variants between the S. cerevisiae and S. paradoxus strains,

458 to avoid re-cutting of the donor allele by Cas 9 after it had been introduced into the recipient

459 strain. We cloned the two guide RNAs, a KANMX cassette, and the gene encoding the $S$.

460 pyogenes Cas9 protein into a single plasmid as described ${ }^{34}$. The resulting plasmid was

461 propagated in DH5a E. coli and miniprepped with a column miniprep kit (Qiagen). Separately, to

462 generate the fragment to be used as the donor for DNA repair after Cas9 cutting, we PCR-

463 amplified the respective region from the donor strain, with primers whose 5'-most 70 base pairs

464 were homologous to the recipient and whose 3'-most 20 base pairs were homologous to the

465 donor, except in the case of transgenesis using S. cerevisiae donors other than DBVPG1373 or

466 S. paradoxus donors other than Z1 (for Figure S10), for which the 3'-most 20 base pairs were

467 homologous to DBVPG1373 or Z1, respectively. The homology region at the 5' end of the gene

468 ended at most 31 base pairs upstream of the 5' cut site, and the homology region at the 3' end

469 of the gene started at most 33 base pairs downstream of the 3 ' cut site. The donor fragment

470 product was purified with a column kit (Qiagen) and ethanol-precipitated. We then

471 simultaneously transformed, using the lithium acetate method, the donor fragment and dual-

472 guide Cas9 plasmid into the recipient strain, using donor:acceptor ratios of $0.38: 10$ to $1: 5$, with

$473 \quad 0.5-26 \mathrm{ug}$ of plasmid. In this transformation, heat shock was for $20-30$ minutes at $39-42^{\circ} \mathrm{C}$ in

474 transformations of S. cerevisiae DBVPG1373, and $10-20$ minutes at $37-39^{\circ} \mathrm{C}$ for transformations 
475 of S. paradoxus Z1. Transformants were plated on YPD+G418 (300 $\mu \mathrm{g} / \mathrm{mL})$ to select for cells

476 that retained the plasmid. From this selection we patched single colonies onto YPD without

477 G418, under the expectation that by the time a lawn came up for each patch, its cells would

478 have lost the Cas9 plasmid. Each such strain was Sanger-sequenced at the junctions of the

479 recipient and donor sequence. Positive patches were streaked to single colonies on YPD plates,

480 and cells from each such colony were used to inoculate a patch on a YPD plate and, separately,

481 to inoculate a patch on a YPD+G418 plate. Those colonies whose patches grew on the former

482 but not on the latter were inferred to be cured of the plasmid and stored at $-80^{\circ} \mathrm{C}$. For all genes

483 except DYN1, 2-3 such strains from each transformation were retained for thermotolerance

484 assays and underwent Sanger sequencing of the entire locus to determine the exact swapped

485 region (Table S1). For DYN1 allele-replacement in S. paradoxus Z1, the Cas9-based strategy

486 yielded a single verified clone in which the $S$. paradoxus Z1 allele of DYN1 was replaced by that

487 of S. cerevisiae DBVPG1373, and likewise for DYN1 allele-replacement in S. cerevisiae

488 DBVPG1373. In each case, we mated the single swap clone to a wild-type of the respective

489 species background, confirmed heterozygosity of the resulting diploid via allele-specific

490 diagnostic PCR at the DYN1 locus, sporulated, and dissected tetrads, allowing each spore to

491 autodiploidize and grow up as a homozygote; we retained from one such tetrad the two spores

492 that were homozygous at the DYN1 locus for the swapped allele, as confirmed by sequencing,

493 and stored these at $-80^{\circ} \mathrm{C}$.

495 Targeted-deletion hemizygote strains for TAF2 were generated by knocking out the S.

496 cerevisiae or the S. paradoxus allele in the interspecific hybrid (JR507) using the above

497 methods for Cas9 cutting and repair, with the following differences. To generate the fragment to

498 be used as the donor for DNA repair after Cas9 cutting, we PCR-amplified the NATMX cassette

499 from pBC713 (a gift from John Dueber, constructed as in ${ }^{35}$ ) using primers whose 5 '-most 70

500 base pairs were homologous to the recipient and whose 3'-most 20 base pairs were 
501 homologous to the cassette. The homology region at the 5' end of the gene ended 22 base

502 pairs outside the 5' cut site (which was upstream of coding start), and the homology region at

503 the 3' end of the gene started 33 base pairs outside the 3' cut site. Positive strains were

504 confirmed by PCR. Two independent transformants were isolated and phenotyped for each

505 genotype (Table S1).

506

507 Growth assays

508

509 Growth measurements of wild-type SGRP strains.

511 For the growth timecourse of a given wild-type, purebred homozygote isolate from the SGRP

512 collection at $28^{\circ} \mathrm{C}$, it was streaked from a $-80^{\circ} \mathrm{C}$ freezer stock onto a YPD agar plate and

513 incubated at $26^{\circ} \mathrm{C}$ for 3 days. A single colony was inoculated into liquid YPD and grown for 24

514 hours at $28^{\circ} \mathrm{C}$ with shaking at $200 \mathrm{rpm}$. This culture was back-diluted into YPD at an $\mathrm{OD}_{600}$ of

$515 \sim 0.05$ and grown for an additional 5.5 hours at $28^{\circ} \mathrm{C}, 200 \mathrm{rpm}$, until reaching logarithmic phase.

516 We transferred cells from each such pre-culture, and YPD, to each of 11 replicate wells of a 96-

517 well plate, with volumes sufficient to yield a total volume of $150 \mu \mathrm{L}$ per well at an $\mathrm{OD}_{600}$ of 0.02 .

518 The plate was covered with a gas-permeable membrane (Sigma) and incubated with orbital

519 shaking in an M200 plate reader (Tecan, Inc.) at $28^{\circ} \mathrm{C}$ for 24 hours. For curves in Figure S1A,

520 measurements for optical density at $595 \mathrm{~nm}\left(\mathrm{OD}_{595}\right)$ were taken every 30 minutes and for each

521 timepoint, the average was taken across replicate wells. To subtract background $\mathrm{OD}_{595}$ for the

522 resulting curve, we tabulated the mean of the five lowest values from all datapoints, excluding

523 the first two, and subtracted this value from that of each timepoint, setting any negative value to

524 0. To smooth the resulting curve, we first replaced each timepoint measurement by its average

525 with those of the timepoints immediately before and after it; then, for any timepoint whose

526 measurement was not greater than or equal to the previous one, we set it to be equal to that 
527 previous data point. For Figure S1B, the efficiency for a given growth curve (from a single well)

528 was calculated as the difference between the $\mathrm{OD}_{595}$ measured at the last four smoothed and

529 averaged data points and that of the first four smoothed and averaged data points. Efficiencies

530 from all of the wells from every S. cerevisiae isolate were combined and compared to

531 efficiencies from all of the wells for every S. paradoxus isolate in a two-sample two-tailed $t$-test.

532

533 For the growth timecourse of a given SGRP strain at $39^{\circ} \mathrm{C}$ (Figure $1 \mathrm{~A}$ ), we used a large-volume

534 flask growth paradigm to avoid the influence of plate effects on growth measurements at high

535 temperature in the incubated microplate reader, as follows. Each strain was streaked from a -

$53680^{\circ} \mathrm{C}$ freezer stock onto a YPD agar plate and incubated at $26^{\circ} \mathrm{C}$ for 3 days. A single colony of a

537 given strain was inoculated into liquid YPD and grown for 24 hours at $28^{\circ} \mathrm{C}$ with shaking at 200

$538 \mathrm{rpm}$. Each of these cultures was back-diluted into YPD at an $\mathrm{OD}_{600}$ of 0.05 and grown for an

539 additional $5.5-7.5$ hours at $28^{\circ} \mathrm{C}$, shaking at $200 \mathrm{rpm}$, until reaching logarithmic phase. We

540 transferred cells from each such pre-culture, and YPD, to a glass $250 \mathrm{~mL}$ flask at the volumes

541 required to attain an $\mathrm{OD}_{600}$ of 0.05 in $100 \mathrm{~mL}$ YPD, and incubated it at $39^{\circ} \mathrm{C}$ with shaking at 200

$542 \mathrm{rpm} . \mathrm{OD}_{600}$ readings were taken every $\sim 2$ hours for $\sim 18$ hours. Figure $1 \mathrm{~A}$ reports representative

543 data from one of three such independent timecourse experiments. For curve fits, we used the

544 getlnitial and SSlogis functions in $\mathrm{R}$ to estimate starting values for the parameters of the logistic

545 equation, and the nls function to fit the final parameters.

546

547 For efficiency measurements of a given SGRP isolate at $39^{\circ} \mathrm{C}$ in the large-volume format

548 (Figure 1B), it was streaked from a $-80^{\circ} \mathrm{C}$ freezer stock onto a YPD agar plate and incubated at

$54926^{\circ} \mathrm{C}$ for 3 days. Two single colonies of each isolate were each inoculated into liquid YPD and

550 grown for 24 hours at $28^{\circ} \mathrm{C}$ with shaking at $200 \mathrm{rpm}$ to generate two replicate pre-cultures. Each

551 was back-diluted into YPD at an $\mathrm{OD}_{600}$ at $600 \mathrm{~nm}$ of 0.05 and grown for an additional 5.5 hours

552 at $28^{\circ} \mathrm{C}$, shaking at $200 \mathrm{rpm}$, until reaching logarithmic phase. The two pre-cultures were each 
553 again back-diluted into YPD in 1-inch diameter glass tubes with a target $\mathrm{OD}_{600}$ of 0.05 ; the

554 actual $\mathrm{OD}_{600}$ of each was measured, after which it was grown at $39^{\circ} \mathrm{C}$ with shaking at $200 \mathrm{rpm}$

555 for 24 hours, and $\mathrm{OD}_{600}$ was measured again. The efficiency for each replicate was calculated

556 as the difference between these final and initial $O D_{600}$ values. The pipeline from inoculation off

557 solid plates through pre-culture, two back-dilutions, and growth at $39^{\circ} \mathrm{C}$ we refer to as a day's

558 growth experiment. For each day's experiments, we calculated the average efficiency across

559 the replicates of each isolate $<e_{\text {strain }}>$. We carried out two days' worth of replicate growth

560 experiments for each isolate. For a given species, we used the complete cohort of

561 measurements of $<e_{\text {strain }}>$ from all isolates of each species, across all days, as input into a two-

562 sample, two-tailed $t$-test to evaluate whether the suite of $e_{\text {strain }}$ values across isolates of $S$.

563 cerevisiae was significantly different from the analogous set of values from S. paradoxus.

565 Growth measurements of targeted-deletion hemizygotes and allele-replacement strains at $28^{\circ} \mathrm{C}$.

567 For efficiency measurements of a given targeted-deletion hemizygote or allele-replacement

568 strain at $28^{\circ} \mathrm{C}$ (Figures S7, S8 and S10), pre-culture and plate reader assays were as for wild-

569 type SGRP strains, except that 6 or more replicate wells were cultured per strain. 2-3

570 independently isolated targeted-deletion hemizygotes or allele-replacement strains (Table S1)

571 were assayed for each genotype. Each timecourse of targeted-deletion hemizygote or allele-

572 replacement strains also included the wild-type hybrid (JR507) or parent (S. cerevisiae

573 DBVPG1373 or S. paradoxus Z1), respectively, with pre-culture and replication as above.

574 Efficiency for a given growth curve (from a single well) was calculated as the difference between

575 the $O D_{600}$ measured at the last four smoothed and averaged datapoints and that of the first four

576 smoothed and averaged datapoints, with smoothing and averaging as detailed above. For

577 Figure S7, relative efficiency for a given well of a given targeted-deletion hemizygote strain at

$57828^{\circ} \mathrm{C}$ was tabulated as its efficiency divided by that of the average of all replicate wells of the 
579 wild-type hybrid (JR507) grown in the same experiment. For a given gene, we used the

580 complete cohort of these measurements, from all isogenic hemizygotes, as input into a two-

581 sample, two-tailed $t$-test to evaluate whether the relative efficiency of the strain in which the $S$.

582 cerevisiae allele was knocked out was lower than the analogous quantity from the strain in

583 which the S. paradoxus allele was knocked out. In Figures S8 and S10, allele-replacement

584 strains for a given gene were analyzed analogously, with the relative efficiency calculated

585 against the respective wild-type parent, and with a one-sample, two-tailed $t$-test to evaluate

586 whether the relative efficiency was significantly different from 1.

Growth measurements of targeted-deletion hemizygotes and allele-replacement strains at $39^{\circ} \mathrm{C}$.

590 For efficiency measurements of a given targeted-deletion hemizygote strain at $39^{\circ} \mathrm{C}$ in the large-

591 volume format (Figure $2 \mathrm{~B}$ ), each strain was streaked from a $-80^{\circ} \mathrm{C}$ freezer stock onto a YPD

592 agar plate and incubated at $26^{\circ} \mathrm{C}$ for 3 days. Two single colonies of a given strain were each

593 inoculated into liquid YPD and grown for 24 hours at $28^{\circ} \mathrm{C}$ with shaking at $200 \mathrm{rpm}$. Each such

594 pre-culture at stationary phase, or a log-phase outgrowth of it (which we used in the case of

595 DYN1 and TAF2: the pre-culture and YPD were added at the volumes required to attain an

$596 \mathrm{OD}_{600}$ of 0.05 and grown for an additional 5.5 hours at $28^{\circ} \mathrm{C}$, shaking at $200 \mathrm{rpm}$, until the

597 culture reached logarithmic phase) was used to inoculate YPD in 1-inch diameter glass culture

598 tubes with a target cell density corresponding to an $\mathrm{OD}_{600}$ of 0.05 . The actual $\mathrm{OD}_{600}$ of each was

599 measured, after which it was grown at $39^{\circ} \mathrm{C}$ with shaking at $200 \mathrm{rpm}$ for 24 hours, and $\mathrm{OD}_{600}$

600 was measured again. The efficiency for each such replicate was then calculated as the

601 difference between the final and initial $O D_{600}$ values. The pipeline from inoculation off solid

602 plates through pre-culture, back-dilution, and growth at $39^{\circ} \mathrm{C}$ we refer to as a day's growth

603 experiment for a targeted-deletion homozygote. In each such experiment, 2-3 independently

604 isolated targeted-deletion hemizygotes of a given gene in each direction were all assayed on 
605 the same day, alongside the wild-type hybrid parent (JR507) with replicate structure and

606 methods as above. From each day's experiments, we calculated the average efficiency across

607 the replicates of the wild-type hybrid $\left\langle e_{\text {hybrid }}>\right.$, and we used this quantity to normalize the

608 efficiency $e_{\text {hemizyg }}$ measured for each replicate of each hemizygote strain assayed on that day.

609 Thus, the final observable used for analysis for each replicate on a given day was

$610 e_{\text {hemizyg }} /<e_{\text {hybrid }}>$. We carried out 2-3 days' replicate growth experiments for each gene's

611 hemizygotes. For a given gene, we used the complete cohort of these measurements of

$612 \mathrm{e}_{\text {hemizyg }} /<\mathrm{e}_{\text {hybrid }}>$, from all days and all isogenic hemizygotes, as input into a two-sample, one-

613 tailed $t$-test to evaluate whether $e_{\text {hemizyg }} /<e_{\text {hybrid }}>$ of the strain in which the $S$. cerevisiae allele

614 was knocked out was lower than the analogous quantity from the strain in which the $S$.

615 paradoxus allele was knocked out.

617 For growth measurements of a given allele-replacement strain at $39^{\circ} \mathrm{C}$ in the large-volume

618 format (Figure 3, S7, S8 and S10), each strain was streaked from a $-80^{\circ} \mathrm{C}$ freezer stock onto a

619 YPD agar plate and incubated at $26^{\circ} \mathrm{C}$ for 3 days. $1-2$ single colonies of each strain were each

620 inoculated into liquid YPD and grown for 24 hours at $28^{\circ} \mathrm{C}$ with shaking at $200 \mathrm{rpm}$ to generate

621 1-2 replicate pre-cultures. Each was back-diluted into YPD at an $\mathrm{OD}_{600}$ of 0.05 and grown for an

622 additional 5.5 hours at $28^{\circ} \mathrm{C}$, shaking at $200 \mathrm{rpm}$, until reaching logarithmic phase. Each pre-

623 culture were each again back-diluted into YPD in 1-inch diameter glass tubes with a target

$624 \mathrm{OD}_{600}$ of 0.05 (for experiments using a single pre-culture, it was now split into two replicate pre-

625 cultures, each of the same $\mathrm{OD}_{600}$ ); the actual $O \mathrm{D}_{600}$ of each was measured, after which it was

626 grown at $39^{\circ} \mathrm{C}$ with shaking at 200 rpm for 24 hours, and $O D_{600}$ was measured again. The

627 efficiency for each replicate was calculated as the difference between these final and initial

$628 \mathrm{OD}_{600}$ values. The pipeline from inoculation off solid plates through pre-culture, two back-

629 dilutions, and growth at $39^{\circ} \mathrm{C}$ we refer to as a day's growth experiment for an allele-swap strain.

630 In each such experiment, 2-3 independently isolated allele-swap strains targeting a given gene 
631 in a given background were all assayed on the same day, alongside the respective wild-type

632 background strain (S. cerevisiae DBVPG1373 or S. paradoxus Z1) with replicate structure and

633 methods as above. For each day's experiments, we calculated the average efficiency across the

634 replicates of the wild-type parent $\left\langle e_{\text {parent }}>\right.$, and we used this quantity to normalize the efficiency

$635 e_{\text {swap }}$ measured for each replicate assayed on that day of each allele-swap strain in the

636 respective background. Thus, the final measurement used for analysis for each replicate on a

637 given day was $\mathrm{e}_{\text {swap }} /<\mathrm{e}_{\text {parent }}>$. We carried out 2-3 days' worth of replicate growth experiments for

638 each gene's allele-swap strains. For a given gene in a given background, we used the complete

639 cohort of measurements of $e_{\text {swap }} /<e_{\text {parent }}>$ from all days and all allele-swap strains as input into a

640 one-sample, one-tailed $t$-test to evaluate whether $\mathrm{e}_{\mathrm{swap}} /<\mathrm{e}_{\text {parent }}>$ was significantly different from

641 1. For swaps of the S. cerevisiae allele of a given gene into S. paradoxus, we tested whether

$642 \mathrm{e}_{\mathrm{swap}} /<\mathrm{e}_{\text {parent }}>$ was greater than 1 (i.e. that the swap strain grew better at $39^{\circ} \mathrm{C}$ than did its

643 parent), and for swaps of the S. paradoxus allele of a given gene into S. cerevisiae, we tested

644 whether $\mathrm{e}_{\text {swap }} /<\mathrm{e}_{\text {parent }}>$ was less than 1 (i.e. that the swap strain grew worse at $39^{\circ} \mathrm{C}$ than its

645 parent).

646

647 Testing survival of wild-type parent strains at $39^{\circ} \mathrm{C}$.

649 To test the survival of heat-treated S. paradoxus Z1 and S. cerevisiae DBVPG1373 (Figure S2),

650 each strain was streaked from $-80^{\circ} \mathrm{C}$ freezer stocks onto YPD agar plates and incubated at

$65126^{\circ} \mathrm{C}$ for 3 days. $1-2$ single colonies of each parent were inoculated into liquid YPD and grown

652 for 24 hours at $28^{\circ} \mathrm{C}$ with shaking at $200 \mathrm{rpm}$ to create 1-2 replicate pre-cultures. After 24 hours,

653 we transferred cells from each pre-culture, and YPD, to each of 2-4 tubes at the volumes

654 required to attain an $\mathrm{OD}_{600}$ of $0.05 \mathrm{in} 11 \mathrm{~mL}$ YPD. Two tubes were incubated at $28^{\circ} \mathrm{C}$ and two at

$65539^{\circ} \mathrm{C}$, all with shaking at $200 \mathrm{rpm}$ for 24 hours. $O D_{600}$ of each was measured; for each culture

656 grown at $28^{\circ} \mathrm{C}, 100 \mu \mathrm{L}$ of a $1.0 \times 10^{-5}$ serial dilution was plated to YPD, and for cultures grown at 
$65739^{\circ} \mathrm{C}$, S. paradoxus and S. cerevisiae were serially diluted to $10^{-1}$ and $5.0 \times 10^{-5}$, respectively.

658 Plates were incubated at $26^{\circ} \mathrm{C}$ for three days until single colonies appeared. Colonies on each

659 plate were counted, from which we tabulated the colony forming units per $\mathrm{mL}$ of culture plated,

660 and normalized by the optical density of the original plated culture. The pipeline from pre-culture

661 through treatment and colony counting we refer to as one day's worth of experiments. We used

662 the results of two days' worth of experiments (a total of four for each species and temperature)

663 as input into an ANOVA with species and temperature as factors, and took the $p$-value for the

664 interaction between the factors as the estimate of the significance of the difference between

665 species in the effect of temperature (of the 24-hour liquid culture) on cells' ability to grow into

666 colonies (at the permissive temperature).

668 Microscopy and quantification

670 For microscopy of S. cerevisiae DBVPG1373 and S. paradoxus Z1 (Figure S3), each species

671 was streaked from a $-80^{\circ} \mathrm{C}$ freezer stock onto a YPD agar plate and incubated at $26^{\circ} \mathrm{C}$ for 3

672 days. Two single colonies of each strain were each inoculated into liquid YPD and grown for 24

673 hours at $28^{\circ} \mathrm{C}$ with shaking at $200 \mathrm{rpm}$ to generate two replicate pre-cultures. Each was then

674 back-diluted into YPD at an $\mathrm{OD}_{600}$ of 0.05 ; one was grown at $39^{\circ} \mathrm{C}$ with shaking at $200 \mathrm{rpm}$ for

67524 hours, and the other was grown at $28^{\circ} \mathrm{C}$ with shaking at $200 \mathrm{rpm}$ for 24 hours. After the 24

676 hour growth period, 0.5 OD units of each culture were harvested through centrifugation and

677 incubated in $66.5 \%$ ethanol for $1-4$ hours at room temperature. Each sample was washed twice

678 with $1 \mathrm{X}$ Dulbecco's Phosphate Buffered Saline (DPBS, Gibco), resuspended in $0.5 \mathrm{~mL}$ of $1 \mathrm{X}$

679 DPBS, and vortexed for 15 seconds on high. $5 \mu \mathrm{L}$ of each sample was transferred to an

680 agarose pad made with 1\% agarose and YPD. We observed samples on a Zeiss Axio Observer

681 inverted bright-field microscope at 100X magnification. Images were taken using a Hamamatsu 
682 ORCA-Flash4.0 digital camera and visualized using ZEN software for image analysis. The

683 exposure of each image was set automatically through ZEN, and brightness was adjusted using

684 the "Min/Max" adjustment for black and white light. The pipeline from inoculation off solid plates

685 through pre-culture, growth at $39^{\circ} \mathrm{C}$ or $28^{\circ} \mathrm{C}$, fixation, and imaging we refer to as a day's

686 experiment. We carried out 2 days' worth of experiments for each species, yielding a total of 17-

68721 images. In each image, free-floating cells were scored manually as singlets or those with a

688 small, medium, or large bud (for a total of 31-151 scored cells per species and temperature).

689 The proportion of scored cells with large buds was tabulated for each day's experiment for each

690 species and temperature. We used these proportions (a total of two for each species and

691 temperature) as input into an ANOVA with species and temperature as factors, and took the $p$ -

692 value for the interaction between the factors as the estimate of the significance of the difference

693 between species in the effect of temperature on the proportion of cells with large buds.

\section{Sequence analysis}

696

$697 D_{x y}$ analysis.

699 To assess the divergence between S. cerevisiae and S. paradoxus at loci mapped by RH-seq,

700 we used the $D_{x y}$ statistic $^{36}$, the average number of pairwise differences between all S. cerevisiae

701 strains and S. paradoxus, normalized for gene length, as follows. We downloaded S. cerevisiae

702 genomic sequences from the following sources: YJM978, UWOPS83-787, Y55, UWOPS05-

703 217.3, 273614N, YS9, BC187, YPS128, DBVPG6765, YJM975, L1374, DBVPG1106, K11,

704 SK1, 378604X, YJM981, UWOPS87-2421, DBVPG1373, NCYC3601, YPS606, Y12,

705 UWOPS05-227.2, and YS2 from http://www.yeastrc.org/g2p/home.do; Sigma1278b, ZTW1, T7, 
706 and YJM789 from http://www.yeastgenome.org/; and RM11 from NCBI (accession

707 PRJNA13674). For each strain, we extracted the coding sequence of each gene in turn, and we

708 downloaded the S. paradoxus reference sequence for each orthologous coding region from ${ }^{17}$.

709 Sequences were aligned using MAFFT ${ }^{37}$ with default settings. Alignments that did not contain a

710 start and stop codon, or those that contained gaps at greater than $40 \%$ of sites were considered

711 poor quality and discarded. We tabulated $D_{x y}$ for each gene. To evaluate whether the eight $\mathrm{RH}-$

712 seq hit genes were enriched for elevated $D_{x y}$, we first tabulated $\left\langle D_{x y}\right\rangle_{\text {true }}$, the mean value across

713 the eight $\mathrm{RH}$-seq hit genes. We then sampled eight random genes from the set of 3416 genes

714 tested by $\mathrm{RH}$-seq; to account for biases associated with lower rates of divergence among

715 essential genes, the resampled set contained six essential genes and two non-essential genes,

716 mirroring the breakdown of essentiality among the $\mathrm{RH}$-seq hits. Across this random sample we

717 tabulated the mean $D_{x y},\left\langle D_{x y}\right\rangle_{\text {resample. }}$ We repeated the resampling 5000 times and used as an

718 empirical $p$-value the proportion of resamples at which $\left\langle D_{x y}\right\rangle_{\text {resample }} \leq\left\langle D_{x y}\right\rangle_{\text {true }}$.

720 Phylogenetic analysis.

722 We downloaded orthologous protein coding regions for the type strains of S. cerevisiae, S.

723 paradoxus, and an outgroup, S. mikatae, from ${ }^{17}$. For each gene for which ortholog sequences

724 were available in all three species, we aligned the sequences with PRANK ${ }^{38}$ utilizing the "-

725 codon" option for codon alignment. These alignments were used as input into the codeml

726 module of $\mathrm{PAML}^{39}$, which was run assuming no molecular clock and allowing omega values to

727 vary for each branch in the phylogeny. From the resulting inferences, we tabulated the branch

728 length on the S. cerevisiae lineage for each gene. To evaluate whether sequence divergence of 
729 the eight $\mathrm{RH}$-seq hit genes showed signatures of rapid evolution along the $\mathrm{S}$. cerevisiae lineage,

730 we used the resampling test detailed above.

732 Locus effect size

734 Locus effect sizes in Figure S9 were computed from the data in Figures 2B and 3 of the main

735 text as follows. For analyses in the hybrid background, for a given locus we calculated $m_{\text {S.par, }}$ the

736 mean of all replicate measurements of $\mathrm{e}_{\text {hemizyg }} /<\mathrm{e}_{\text {hybrid }}>$ of hemizygote strains lacking the $S$.

737 paradoxus allele. We took, as independent estimates of the locus effect size (each

738 corresponding to an open circle on the respective grey bar in Figure S9), each measurement of

$739 \mathrm{e}_{\text {hemizyg }} /<\mathrm{e}_{\text {hybrid }}>$ of a hemizygote strain lacking the $S$. cerevisiae allele, as a ratio against $m_{\text {S.par. }}$.

740 For analyses in the S. cerevisiae DBVPG1373 background, for a given locus we used each

741 measurement of $e_{\text {swap }} /<e_{\text {parent }}>$ as an independent estimate of the locus effect size (each

742 corresponding to an open circle on the respective orange bar in Figure S9). For analyses in the

743 S. paradoxus Z1 background, for a given locus we used each measurement of $<e_{\text {parent }}>/ e_{\text {swap }}$ as

744 an independent estimate of the locus effect size (each corresponding to an open circle on the

745 respective blue bar in Figure S9).

747 Data availability

748

749 RH-seq data will be deposited in the Sequence Read Archive (SRA) upon publication.

\section{Code availability}


bioRxiv preprint doi: https://doi.org/10.1101/220913; this version posted June 14, 2018. The copyright holder for this preprint (which was not certified by peer review) is the author/funder. All rights reserved. No reuse allowed without permission.

753 Custom Python and $\mathrm{R}$ scripts used for $\mathrm{RH}$-seq data analysis are available upon request.

754 


\section{References}

756

757

758

759

760

761

762

763

764

765

766

767

768

769

770

771

772

773

774

775

776

777

778

779

780

781

782

783

784

785

786

787

788

789

790

791

792

793

794

795

796

797

798

799

800

801

802
1 Flint, J. \& Mott, R. Finding the molecular basis of quantitative traits: successes and pitfalls. Nat Rev Genet 2, 437-445, doi:10.1038/35076585 (2001).

2 Good, B. H., McDonald, M. J., Barrick, J. E., Lenski, R. E. \& Desai, M. M. The dynamics of molecular evolution over 60,000 generations. Nature, doi:10.1038/nature24287 (2017).

3 Savolainen, O., Lascoux, M. \& Merila, J. Ecological genomics of local adaptation. Nat Rev Genet 14, 807-820, doi:10.1038/nrg3522 (2013).

$4 \quad$ Allen Orr, $\mathrm{H}$. The genetics of species differences. Trends Ecol Evol 16, 343-350 (2001).

5 Nadeau, N. J. \& Jiggins, C. D. A golden age for evolutionary genetics? Genomic studies of adaptation in natural populations. Trends Genet 26, 484-492, doi:10.1016/j.tig.2010.08.004 (2010).

6 Wray, G. A. Genomics and the Evolution of Phenotypic Traits. Annual Review of Ecology, Evolution, and Systematics 44, 51-72 (2013).

7 Masly, J. P. \& Presgraves, D. C. High-resolution genome-wide dissection of the two rules of speciation in Drosophila. PLoS biology 5 , e243, doi:10.1371/journal.pbio.0050243 (2007).

8 Greig, D. A screen for recessive speciation genes expressed in the gametes of F1 hybrid yeast. PLoS genetics 3, e21, doi:10.1371/journal.pgen.0030021 (2007).

9 Eshed, Y. \& Zamir, D. An introgression line population of Lycopersicon pennellii in the cultivated tomato enables the identification and fine mapping of yield-associated QTL. Genetics 141, 1147-1162 (1995).

10 Lazzarano, S. et al. Genetic mapping of species differences via in vitro crosses in mouse embryonic stem cells. Proc Natl Acad Sci U S A 115, 3680-3685, doi:10.1073/pnas.1717474115 (2018).

11 Roop, J. I., Chang, K. C. \& Brem, R. B. Polygenic evolution of a sugar specialization trade-off in yeast. Nature 530, 336-339, doi:10.1038/nature16938 (2016).

12 Steinmetz, L. M. et al. Dissecting the architecture of a quantitative trait locus in yeast. Nature 416, 326-330, doi:10.1038/416326a (2002).

13 Stern, D. L. Identification of loci that cause phenotypic variation in diverse species with the reciprocal hemizygosity test. Trends Genet 30, 547-554, doi:10.1016/j.tig.2014.09.006 (2014).

14 Goncalves, P., Valerio, E., Correia, C., de Almeida, J. M. \& Sampaio, J. P. Evidence for divergent evolution of growth temperature preference in sympatric Saccharomyces species. PLoS One 6, e20739, doi:10.1371/journal.pone.0020739 (2011).

15 Salvado, Z. et al. Temperature adaptation markedly determines evolution within the genus Saccharomyces. Appl Environ Microbiol 77, 2292-2302, doi:10.1128/AEM.0186110 (2011).

16 Sweeney, J. Y., Kuehne, H. A. \& Sniegowski, P. D. Sympatric natural Saccharomyces cerevisiae and S. paradoxus populations have different thermal growth profiles. FEMS Yeast Res 4, 521-525 (2004).

17 Scannell, D. R. et al. The Awesome Power of Yeast Evolutionary Genetics: New Genome Sequences and Strain Resources for the Saccharomyces sensu stricto Genus. G3 (Bethesda) 1, 11-25, doi:10.1534/g3.111.000273 (2011).

18 Hartwell, L. H. Saccharomyces cerevisiae cell cycle. Bacteriol Rev 38, 164-198 (1974).

19 Mitra, R., Fain-Thornton, J. \& Craig, N. L. piggyBac can bypass DNA synthesis during cut and paste transposition. EMBO J 27, 1097-1109, doi:10.1038/emboj.2008.41 (2008). 
80320 van Opijnen, T., Lazinski, D. W. \& Camilli, A. Genome-Wide Fitness and Genetic

804

805

806

807

808

809

810

811

812

813

814

815

816

817

818

819

820

821

822

823

824

825

826

827

828

829

830

831

832

833

834

835

836

837

838

839

840

841

842

843

844

845

846

847

848

849

850

851

852

Interactions Determined by Tn-seq, a High-Throughput Massively Parallel Sequencing Method for Microorganisms. Curr Protoc Mol Biol 106, 716 11-24, doi:10.1002/0471142727.mb0716s106 (2014).

21 Parts, L. et al. Revealing the genetic structure of a trait by sequencing a population under selection. Genome Res 21, 1131-1138, doi:10.1101/gr.116731.110 (2011).

22 Winzeler, E. A. et al. Functional characterization of the S. cerevisiae genome by gene deletion and parallel analysis. Science 285, 901-906 (1999).

23 Leuenberger, $\mathrm{P}$. et al. Cell-wide analysis of protein thermal unfolding reveals determinants of thermostability. Science 355, doi:10.1126/science.aai7825 (2017).

24 Wilkening, S. et al. An evaluation of high-throughput approaches to QTL mapping in Saccharomyces cerevisiae. Genetics 196, 853-865, doi:10.1534/genetics.113.160291 (2014).

25 Neurological Disorders: Public Health Challenges. 232 (World Health Organization, 2006).

26 Sinha, H., Nicholson, B. P., Steinmetz, L. M. \& McCusker, J. H. Complex genetic interactions in a quantitative trait locus. PLoS Genet 2, e13, doi:10.1371/journal.pgen.0020013 (2006).

27 Guldener, U., Heck, S., Fielder, T., Beinhauer, J. \& Hegemann, J. H. A new efficient gene disruption cassette for repeated use in budding yeast. Nucleic Acids Res 24, 25192524 (1996).

28 Wetmore, K. M. et al. Rapid quantification of mutant fitness in diverse bacteria by sequencing randomly bar-coded transposons. MBio 6, e00306-00315, doi:10.1128/mBio.00306-15 (2015).

29 Skelly, D. A. et al. Integrative phenomics reveals insight into the structure of phenotypic diversity in budding yeast. Genome Res 23, 1496-1504, doi:10.1101/gr.155762.113 (2013).

30 Langmead, B. \& Salzberg, S. L. Fast gapped-read alignment with Bowtie 2. Nat Methods 9, 357-359, doi:10.1038/nmeth.1923 (2012).

31 Liti, G. et al. Population genomics of domestic and wild yeasts. Nature 458, 337-341, doi:10.1038/nature07743 (2009).

$32 \mathrm{Li}, \mathrm{H}$. et al. The Sequence Alignment/Map format and SAMtools. Bioinformatics 25, 2078-2079, doi:10.1093/bioinformatics/btp352 (2009).

33 Roop, J. I. \& Brem, R. B. Rare variants in hypermutable genes underlie common morphology and growth traits in wild Saccharomyces paradoxus. Genetics 195, 513-525, doi:10.1534/genetics.113.155341 (2013).

34 Maurer, M. J. et al. Quantitative Trait Loci (QTL)-Guided Metabolic Engineering of a Complex Trait. ACS Synth Bio/ 6, 566-581, doi:10.1021/acssynbio.6b00264 (2017).

35 Lee, M. E., DeLoache, W. C., Cervantes, B. \& Dueber, J. E. A Highly Characterized Yeast Toolkit for Modular, Multipart Assembly. ACS Synth Biol 4, 975-986, doi:10.1021/sb500366v (2015).

36 Nei, M. Molecular Evolutionary Genetics. (Columbia University Press, 1987).

37 Katoh, K. \& Standley, D. M. MAFFT multiple sequence alignment software version 7: improvements in performance and usability. Mol Biol Evol 30, 772-780, doi:10.1093/molbev/mst010 (2013).

38 Loytynoja, A. \& Goldman, N. webPRANK: a phylogeny-aware multiple sequence aligner with interactive alignment browser. BMC Bioinformatics 11, 579, doi:10.1186/1471-210511-579 (2010).

39 Yang, Z. PAML 4: phylogenetic analysis by maximum likelihood. Mol Biol Evol 24, 15861591, doi:10.1093/molbev/msm088 (2007). 


\section{End Notes}

854 Supplementary Information is available in the online version of the paper.

855

856 Acknowledgements The authors thank Faisal AlZaben, Anna Flury, Gina Geiselman,

857 Justin Hong, Jake Kim, Matt Maurer, and Luke Oltrogge for technical assistance, Dave

858 Savage for his generosity with microscopy resources, and Ben Blackman, Sam

859 Coradetti, Avi Flamholz, Vinny Guacci, Doug Koshland, Chris Nelson, and Arjun

860 Sasikumar for discussions. This work was supported by R01 GM120430-A1 and by

861 Community Sequencing Project 1460 to RBB at the U.S. Department of Energy Joint

862 Genome Institute, a DOE Office of Science User Facility. The work conducted by the

863 latter was supported by the Office of Science of the U.S. Department of Energy

864 under Contract No. DE-AC02-05CH11231.

865

866 Author Contributions R.B.B. and J.I.R. developed the project design; C.V.W., J.I.R.,

867 R.H. and J.C. performed experiments; C.V.W. and J.I.R. and analyzed the data; J.M.S.

868 contributed to the development of mutagenesis and sequencing methods; A.P.A.

869 contributed mutagenesis and sequencing reagents; I.V.G. provided technical assistance

870 with sequencing; R.B.B. and C.V.W wrote the manuscript with input from all authors.

871

872 Author Information The authors do not declare any competing financial interests.

873 Correspondence and request for materials should be addressed to R.B.B.

874 (rbrem@buckinstitute.org) and J.I.R. (jeremyianroop@gmail.com). 